\title{
Interfacial water reorganization as a pH-dependent descriptor of the hydrogen evolution rate on platinum electrodes
}

\author{
I. Ledezma-Yanez', W.D.Z. Wallace'1, P. Sebastián-Pascual², V. Climent ${ }^{2}$, J.M. Feliu², M.T.M. \\ Koper $^{1}$ \\ ${ }^{1}$ Leiden Institute of Chemistry, Leiden University, 2300 RA, Leiden, The Netherlands \\ ${ }^{2}$ Instituto de Electroquímica, Universidad de Alicante, Apdo. 99, Alicante, Spain \\ E-mail: $\underline{\text { m.koper@chem.leidenuniv.nl }}$
}

\begin{abstract}
The hydrogen evolution on platinum is a milestone reaction in electrocatalysis as well as an important reaction towards sustainable energy storage. Remarkably, the $\mathrm{pH}$ dependent kinetics of this reaction is not yet fully understood. Here, we present a detailed kinetic study of the hydrogen adsorption and evolution reaction on $\mathrm{Pt}(111)$ in a wide $\mathrm{pH}$ range. Impedance and Tafel slope measurements show that the hydrogen adsorption and hydrogen evolution are both slow in alkaline media, which is consistent with the observation of a shift in the rate-determining step for $\mathrm{H}_{2}$ evolution. Adding nickel to the $\mathrm{Pt}(111)$ surface lowers the barrier for the hydrogen adsorption rate in alkaline solutions and thereby enhances the hydrogen evolution rate. These observations are explained by a new model which highlights the role of the reorganization of interfacial water to accommodate charge transfer through the electric double layer, the energetics of which is controlled by how strongly water interacts with the interfacial field. The new model is supported by laser-induced temperature-jump measurements. Our model sheds new light on the origin of the slow kinetics for the hydrogen evolution reaction in alkaline media.
\end{abstract}

\section{Introduction}

There is a global call for industrial processes that combine economic progress with long-term preservation of natural resources. In terms of technological advances for sustainable energy production ${ }^{1,2}$, there is a recent renewed interest to realize the so- 
called hydrogen economy ${ }^{3,4}$ by photocatalytic water splitting or by the combination of photovoltaics with water electrolysis ${ }^{5,6}$. Methane steam-reforming ${ }^{7}$ is currently the most cost-efficient technology available for hydrogen production, but unsustainable in the long run as it is still based on the deployment of fossil fuels. In order for the hydrogen economy to meet our future energy demands ${ }^{8}$, there are, however, various fundamental bottlenecks to be overcome, such as that related to the efficient catalysis of the associated multi-proton multi-electron transfer reactions. Essentially, significant advances are required to lower the high inherent costs of electrocatalysts, by increasing the efficiency of water oxidation, by the replacement of scarce and expensive catalyst materials by earth-abundant alternatives, and by maximizing their durability and lifetime. Substantial efforts have thus been devoted to lowering the costs of the electrodes necessary for water splitting ${ }^{9,10}$. Recent theoretical works exposed the mechanistic features of the oxygen evolution reaction (OER $)^{11,12}$, taking place at the anodes of electrolyzers, in which water is oxidized to produce molecular oxygen and protons. These insights motivated several reports on earth-abundant and highly-efficient OER anode materials that typically work at high $\mathrm{pH}^{13,14}$. Unfortunately, the price to be paid for the use of alkaline conditions is a significant overpotential at the cathode, where the hydrogen evolution reaction (HER) takes place.

It has long been known in the electrochemistry literature that the kinetics of both the hydrogen evolution reaction (HER) and hydrogen oxidation reaction (HOR) on platinum are significantly slower in alkaline media than in acidic media ${ }^{15-17}$. The elucidation of the molecular-level origin of this problem would be of obvious importance for the further development of alkaline electrolyzers and alkaline fuel cells. Marković et al. have recently shown ${ }^{18-20}$ how the oxophilicity of the interface, as modified by adsorbing a small amount of $\mathrm{Ni}(\mathrm{OH})_{2}$ on $\mathrm{Pt}(111)$, may improve the kinetics of the $\mathrm{HER} / \mathrm{HOR}$, and ascribed this effect to the favorable interaction of surface adsorbed $\mathrm{OH}_{\text {ads }}$ with the relevant intermediates. Mechanistic studies on the HER ${ }^{18,19,21-24}$ have traditionally correlated reaction rates with thermodynamic descriptors, in particular the strength of the bond between hydrogen and the metal electrode, following the Sabatier principle ${ }^{25,26}$. Gasteiger et al. and Yan et al. have suggested ${ }^{22,27}$ that a $\mathrm{pH}$-dependent $\mathrm{H}$-binding energy lies at the origin of the $\mathrm{pH}$ dependent HER/HOR kinetics, and therefore they concluded that the $\mathrm{H}$-binding energy is and remains the sole descriptor for the HER/HOR reaction. Specifically, 
Yan et al. have considered the $\mathrm{pH}$-dependent shifts of the voltammetric peaks in the so-called "underpotential deposition" (UPD) hydrogen region of polycrystalline platinum as evidence for such a $\mathrm{pH}$-dependent $\mathrm{H}$-binding energy. However, the molecular-level origin of the $\mathrm{pH}$-dependent $\mathrm{H}$-binding energy has remained elusive. More importantly, we have recently argued ${ }^{28}$ that the nature of the "hydrogen" peaks on polycrystalline platinum are unlikely to be associated with the adsorption of hydrogen only, but also include the effect of the adsorption of oxygenated species on (110) and (100) sites. Therefore, their peak potentials are not unambiguous indicators of $\mathrm{H}$-binding energy. Additionally, on a $\mathrm{Pt}(111)$ electrode, there is no significant shift of the H-UPD with $\mathrm{pH}$, but there is still a very significant $\mathrm{pH}$ dependence of the HER/HOR kinetics (see below). Therefore, it appears that in spite of the undeniable success of the traditional models, they overlook important kinetic details, and that a consistent explanation of why HER/HOR on Pt is slow in alkaline media is still missing.

In this work, we present a detailed kinetic study of the hydrogen adsorption and evolution reaction on a $\mathrm{Pt}(111)$ single-crystal electrode, in a wide $\mathrm{pH}$ range, and in the absence and presence of a small amount of $\mathrm{Ni}(\mathrm{OH})_{2}$ promoter. Electrochemical impedance spectroscopy is used to accurately measure the charge transfer kinetics of hydrogen adsorption. We present a new model for the rate of hydrogen adsorption step, based on the idea that the barrier for this reaction depends on how close or remote the electrode potential is in relation to the potential of zero (free) charge (pzfc). The laser-induced temperature jump technique is then used to measure the potential of maximum entropy (pme), which is closely related to the pzfc, to show that at $\mathrm{pH} 13$, the addition of $\mathrm{Ni}(\mathrm{OH})_{2}$ to $\mathrm{Pt}(111)$ indeed shifts the pme/pzfc closer to the hydrogen adsorption region. We attribute the impact of the pzfc on the activation barrier of hydrogen adsorption to the energy penalty associated with the reorganization of interfacial water to accommodate charge movement through the double layer. In acid media, the pzc/pme of $\mathrm{Pt}(111)$ is close to the hydrogen region, and the energy of reorganization of the interfacial water to move a proton through the double layer is relatively small. In alkaline media, the pzc/pme of $\mathrm{Pt}(111)$ is far from the hydrogen region (i.e. close to the $\mathrm{OH}_{\text {ads }}$ region) and the corresponding strong electric field existing at the electrode/electrolyte interface in the hydrogen region leads to a large reorganization energy for interfacial water when $\mathrm{OH}^{-}$transfers 
through the double layer. In general terms, our results show how a cost-effective, earth-abundant metal as nickel, in the form of nickel hydroxide, promotes the reorganization of water networks at the electrode-electrolyte interface by shifting the pzc closer to the equilibrium potential of the HER reaction, thereby enhancing the reaction rate for the hydrogen evolution at high $\mathrm{pH}$ values. Our model also suggests a new strategy for designing new and better electrocatalysts in aqueous media, highlighting the role of the interfacial solvent reorganization on ion transfer steps.

\section{Results and Discussion}

\section{Kinetic measurements on $\mathrm{H}$ adsorption and $\mathrm{H}_{2}$ evolution as a function of $\mathrm{pH}$}

Figure 1 illustrates the key observation that we wish to explain in this work, comparing the cyclic voltammetries for a $\mathrm{Pt}(111)$ electrode at pH 11 (blue line), $\mathrm{pH} 12$ (red line), $\mathrm{pH} 13$ (turquoise line) and pH 1 (black, dashed line). All the measurements were collected using a scan rate of $50 \mathrm{mV}^{-\mathrm{s}^{-1}}$. The voltammetric curves are very similar in current and shape for the alkaline $\mathrm{pH}$, showing the typical features for the $\mathrm{Pt}$ (111) surface orientation: the hydrogen adsorption region, known as H-UPD (0.1 $0.35 \mathrm{~V}_{\mathrm{RHE}}$ ), which remains almost unaltered for the several $\mathrm{pH}$ values presented (versus the RHE), the double layer region (0.35 - $0.55 \mathrm{~V}_{\mathrm{RHE}}$ ), and the hydroxyl adsorption-desorption region $\left(0.6-0.8 \mathrm{~V}_{\mathrm{RHE}}\right)$. The reduction current registered between $0.05 V_{\text {RHE }}$ and $-0.1 V_{R H E}$ corresponds to the hydrogen evolution, and the adsorbed hydrogen formed in this potential window is referred to as H-OPD (overpotential deposition). We observe that the onset potential for the HER in alkaline solutions exhibits a shift towards negative potentials as the $\mathrm{pH}$ value increases. Hydrogen evolution clearly has the lowest overpotential at $\mathrm{pH} 1$, even though there is only a small shift in the H-UPD region in comparison to alkaline media. We conclude from Figure 1 that a $\mathrm{pH}$-dependent shift in the $\mathrm{H}$-binding energy (if any) cannot explain the significant $\mathrm{pH}$ dependence of the HER overpotential on $\mathrm{Pt}(111)$. 


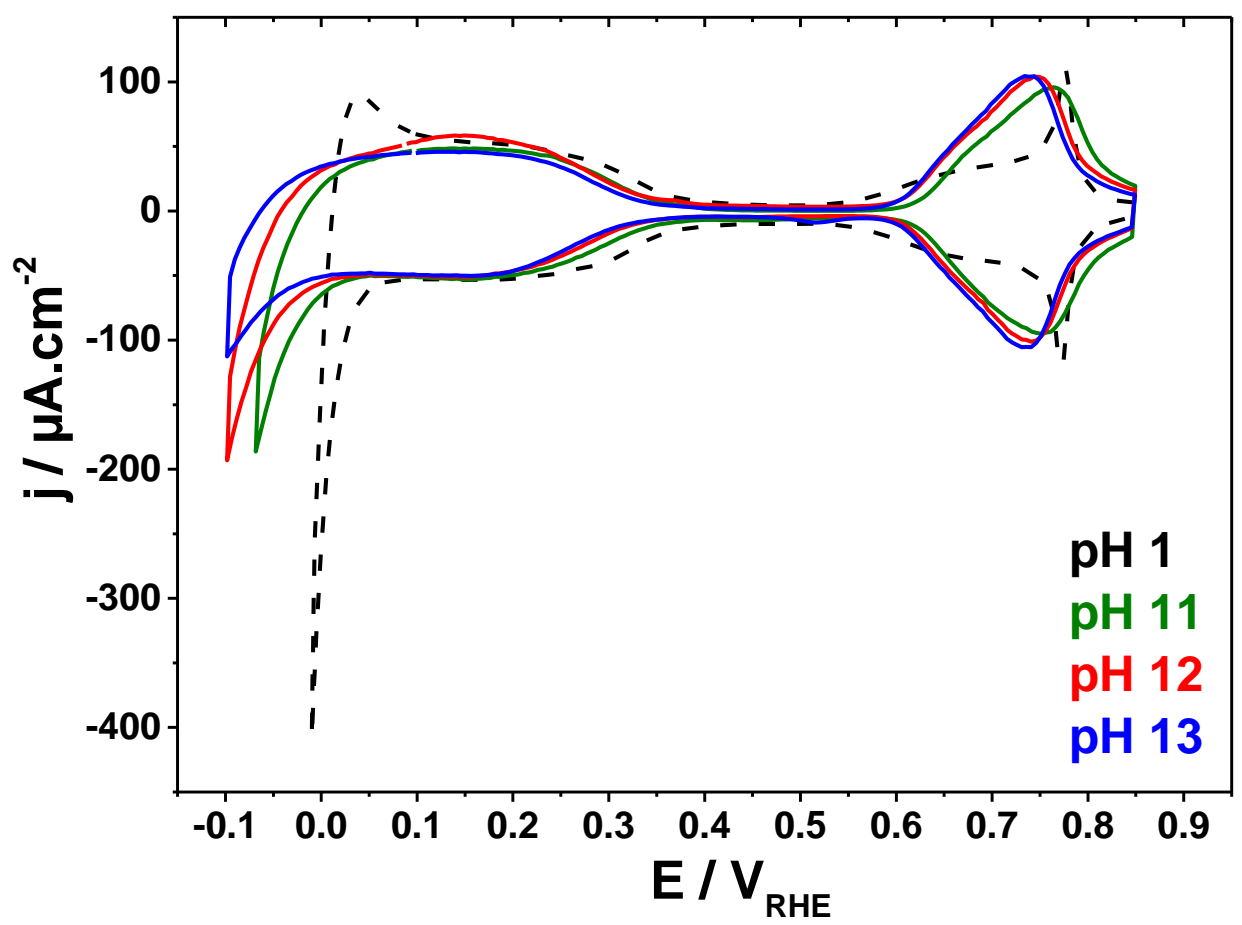

Figure 1. Cyclic voltammetries for $\mathrm{Pt}(111)$ in $0.1 \mathrm{M}$ perchloric acid (pH 1); $0.001 \mathrm{M} \mathrm{NaOH}+0.099 \mathrm{M} \mathrm{NaClO}_{4}(\mathrm{pH} 11) ; 0.01 \mathrm{M}$ $\mathrm{NaOH}+0.09 \mathrm{M} \mathrm{NaClO}_{4}(\mathrm{pH} 12) ; 0.1 \mathrm{M} \mathrm{NaOH}$ (pH 13). Scan rate: $50 \mathrm{mV} . \mathrm{s}^{-1}$.

To demonstrate that the $\mathrm{pH}$ also affects the rate of hydrogen adsorption in the $\mathrm{H}$ UPD region, we studied the kinetics of hydrogen adsorption in the 0.1-0.3 V potential window. Figure 2 summarizes the measurements made by means of electrochemical impedance spectroscopy (EIS) as a function of potential and $\mathrm{pH}$. The experimental admittance spectra were fitted using the equivalent electric circuit (EEC 17,29) presented in Figure 2a. This EEC corresponds to a simple heterogeneous adsorption step, forming an adsorbed intermediate without diffusion limitation. The charge transfer resistance, $R_{c t}$, is inversely proportional to the rate of the corresponding hydrogen adsorption reaction, i.e. $\mathrm{H}^{+}+\mathrm{e}^{-} \rightarrow \mathrm{H}_{\text {ads }}$ in acidic media, and $\mathrm{H}_{2} \mathrm{O}+\mathrm{e}^{-} \rightarrow$ $\mathrm{H}_{\text {ads }}+\mathrm{OH}^{-}$in alkaline media. Rsol stands for the resistance of the solution, whereas $C D L$ and $C_{A D}$ represent the capacitance of the double layer and the pseudocapacitance of the adsorbed hydrogen, respectively. Figure $2 b$ shows the admittance plots, measured at $0.1 \mathrm{~V}_{\mathrm{RHE}}$ in solutions of $0.1 \mathrm{M}$ ionic strength at different $\mathrm{pH}$ values. The data collected for the entire H-UPD region were fitted to the EEC shown in Figure $2 \mathrm{a}$ and can be found in Supplementary Figure 1. From the Nyquist plots presented in Figure $2 \mathrm{~b}$ we notice that for alkaline $\mathrm{pH}$ we observe two semicircles. 
The first semicircle at low frequencies (left side of each diagram) is related to the charge transfer resistance. For acidic media, only a single semicircle in the admittance plane is observed in our measurements (not shown, see ref. 26), meaning that the H-UPD charge transfer process is too fast to be measured in acidic media ${ }^{17}$. These experimental observations reveal that not only the HER and the associated H-OPD formation is slower in alkaline media, but also the H-UPD formation is slower, even if the thermodynamic driving force for hydrogen adsorption is the same (as all potentials are referred to the RHE scale and there is no observable $\mathrm{pH}$ dependence of the $\mathrm{H}$-binding energy on $\mathrm{Pt}(111)$ ).

The $R_{c t}$ measurements were performed for solutions with different $\mathrm{pH}$ value for the $\mathrm{H}$ UPD region and are shown in Figure $2 \mathrm{c}$. The $\mathrm{R}_{\mathrm{ct}}$ for $\mathrm{pH} 7$ is shown for comparison with the alkaline $\mathrm{pH}$ values $\mathrm{pH} 7$ was measured in a phosphate buffer, but since there is no phosphate adsorption in the H-UPD region ${ }^{30}$, we do not expect an impact of phosphate on the hydrogen adsorption rate). From the figure we observe that the charge transfer resistance is both $\mathrm{pH}$ - and potential-dependent. Charge transfer resistances are obtained from fits of the data in Supplementary Figure 1 by a mathematical model derived for the circuit in Figure $2 \mathrm{a}^{29,31}$. The mathematical expression for charge transfer resistance includes the rate constant for hydrogen adsorption, which has a Butler-Volmer-type potential dependence; hence, the charge transfer resistance increases with more positive potential, as has been analysed in detail elsewhere ${ }^{17}$. The reaction becomes slower for higher $\mathrm{pH}$ values, suggesting that the reaction barrier for hydrogen adsorption increases with $\mathrm{pH}$. This effect cannot be described in terms of a presumed $\mathrm{pH}$-dependent $\mathrm{H}$-binding energy and must therefore have another origin. 

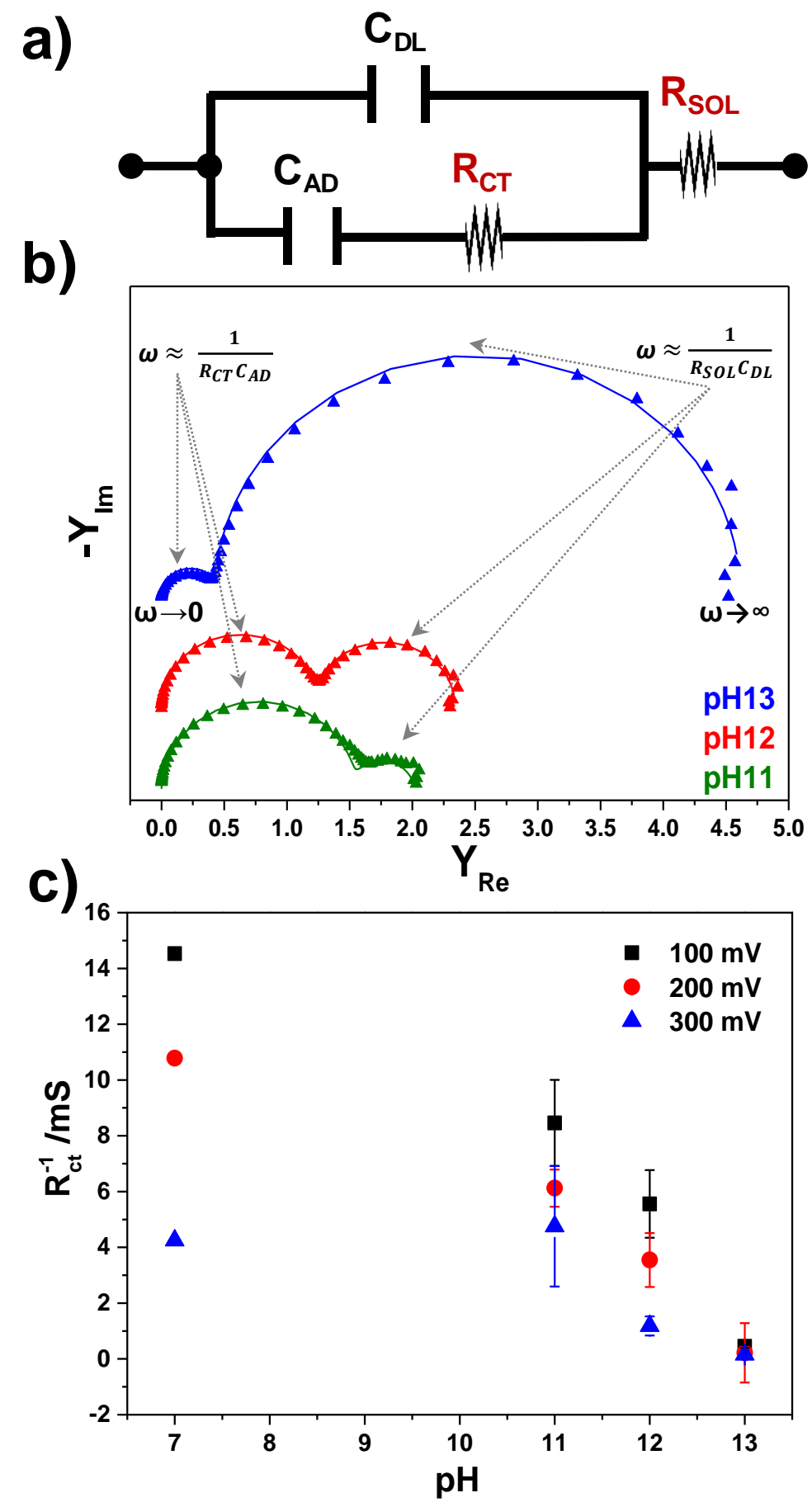

Figure 2. a) Equivalent electric circuit for H-UPD region, featuring the double layer capacitance, $\mathrm{C}_{\mathrm{DL}}$, the capacitance associated to the hydrogen adsorption, $\mathrm{C}_{\mathrm{AD}}$, the charge-transfer resistance for hydrogen adsorption, $\mathrm{R}_{\mathrm{CT}}$, and the solution resistance, $R_{s o L}$. b) Admittance Nyquist plots for $\mathrm{Pt}(111)$ in $0.1 \mathrm{M}$ solutions for different $\mathrm{pH}$ values, measured with frequencies ranging from $10 \mathrm{kHz}$ to $0.1 \mathrm{~Hz}$ and an amplitude of $5 \mathrm{mV}$, at $0.2 \mathrm{~V}_{\mathrm{RHE}}$ : the dots represent the experimental data points collected, while the solid lines correspond to the fit obtained using the EEC. The arrows point out how the components from the EEC in Fig. $2 \mathrm{a}$ are related to each semi-circle. The data for the entire H-UPD region can be found in Figure Supplementary S1. c) Inverse of the charge transfer resistance as a function of the $\mathrm{pH}$ value, proving that the rate of H-UPD formation becomes slower in more alkaline solutions. The $\mathrm{R}_{\mathrm{ct}}$ was obtained from the fitting parameters for the admittance plots, collected for $\mathrm{Pt}(111)$ in $0.1 \mathrm{M}$ solutions with different $\mathrm{pH}$ value. The different colors represent the potential at which the data was collected (see 
legend), whereas the error bars correspond to the standard deviation of three measurements. The impedance at $\mathrm{pH}=7$ was measured only once.

The mechanism for the hydrogen evolution at each $\mathrm{pH}$ value can be derived from the relationship between the current density and overpotential, known as Tafel plots. In order to estimate the Tafel slopes, we recorded polarization curves at $10 \mathrm{mV} . \mathrm{s}^{-1}$ under argon atmosphere, using $0.1 \mathrm{M}$ solutions with various $\mathrm{pH}$ values between 1 and 13, as shown in Supplementary Figures 2 and 3 We find that on $\operatorname{Pt}(111)$ the Tafel slope increases with $\mathrm{pH}$, as reported previously ${ }^{16,32,33}$ for pre-activated polycrystalline platinum electrodes. The significant conclusion from the combination of Figures 1, 2 and the Tafel slope measurements is that not only the rates of HER and H-UPD on $\mathrm{Pt}(111)$ change with $\mathrm{pH}$, but also the HER mechanism.

The relation of the rate-determining step in the mechanism to Tafel slope analysis has been described elsewhere ${ }^{34}$. Experimental reports ${ }^{33,35,36}$ on polycrystalline and low-index single-crystal platinum electrodes under hydrodynamic conditions, exhibit

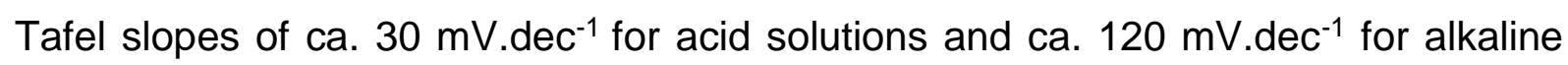
solutions. Previous reported values of the Tafel slope for HER in acidic media are 31 $\mathrm{mV} . \mathrm{dec}^{-1}$ in perchloric acid by Seto et al. ${ }^{35}$ and $35 \mathrm{mV} \cdot \mathrm{dec}^{-1}$ in sulphuric acid by Kita et al. ${ }^{37}$ The interpretation of the measured Tafel slope is as follows. A value around $120 \mathrm{mV} \cdot \mathrm{dec}^{-1}$ implies that the first electron-transfer step (ET) is rate-determining (RDS), meaning that in alkaline media the so-called Volmer step $\mathrm{H}_{2} \mathrm{O}+\mathrm{e}^{-} \rightarrow \mathrm{H}_{\text {ads }}+$ $\mathrm{OH}^{-}$is the slowest reaction step. A Tafel slope of ca. $40-30 \mathrm{mV} \cdot \mathrm{dec}^{-1}$ implies that either the second ET step is the RDS or the RDS is a chemical step preceded by two ET steps, indicating that in acidic media the mechanism involves the Volmer step $\mathrm{H}^{+}$ $+\mathrm{e}^{-} \leftrightarrow \mathrm{H}_{\text {ads }}$ in equilibrium, followed by a rate-determining Tafel step $2 \mathrm{H}_{\text {ads }} \rightarrow \mathrm{H}_{2}$ or a rate-determining Heyrovsky step $\mathrm{H}^{+}+\mathrm{e}^{-}+\mathrm{H}_{\text {ads }} \rightarrow \mathrm{H}_{2}$. These mechanistic implications are consistent with the observation that in alkaline solutions the hydrogen adsorption reaction is a slow reaction, whereas in acidic media this step is fast and in equilibrium. The experiments in Figures 1 and 2 were performed in the absence of $\mathrm{H}_{2}$ gas in solution, as we aim to study only the hydrogen adsorption and hydrogen evolution reactions. The fast and slow kinetics and the corresponding Tafel slopes measured for the HER at $\mathrm{pH}=1$ and $\mathrm{pH}=13$ are independent of whether the experiment is performed in Argon or $\mathrm{H}_{2}$ saturated solution (see Supplementary Figure 4). 
Figure 3 compares the rate of hydrogen adsorption, as evaluated by EIS, and the rate of hydrogen evolution at $\mathrm{pH} 13$ on bare $\mathrm{Pt}(111)$ and on $\mathrm{Pt}(111)$ in presence of a small amount of $\mathrm{Ni}(\mathrm{OH})_{2}$. The Tafel plots for the HER (Fig. 3a) and the inverse of the $\mathrm{R}_{\mathrm{ct}}$ for H-UPD (Fig. 3b) for $\mathrm{Pt}(111) / \mathrm{Ni}(\mathrm{OH})_{2}$ show a clear enhancement of both rates in alkaline media, as compared to $\mathrm{Pt}(111)$. The HER rate enhancement by $\mathrm{Ni}(\mathrm{OH})_{2}$ in Fig.3a was already reported in previous works ${ }^{18,20}$ by Marković et al.; the novelty here is that this enhancement also applies to the rate of H-UPD formation (Fig.3b). From the Tafel plots in Figure 3a we can observe that the rate for hydrogen evolution in the presence of $\mathrm{Ni}(\mathrm{OH})_{2}$ is now in between that for $\mathrm{pH} 13$ and $\mathrm{pH} 1$ on pure $\mathrm{Pt}(111)$, with a corresponding change in Tafel slope. Together with the data in Fig.3b, this suggests that the role of $\mathrm{Ni}(\mathrm{OH})_{2}$ is to lower the barrier for the hydrogen adsorption reaction, rather than to change the energetics of the hydrogen intermediate or the mechanism.
a)
HER
b) H-UPD
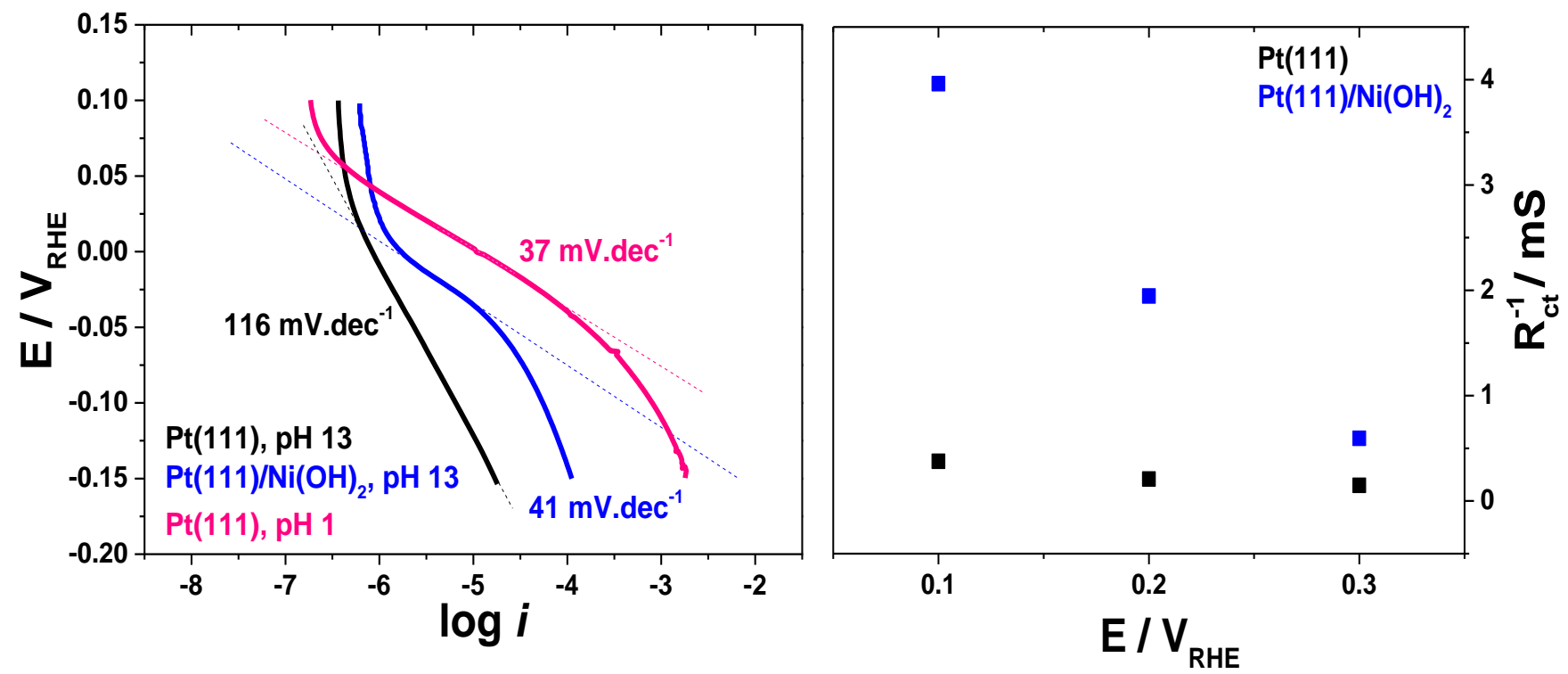

Figure 3. a) Tafel plots for $\mathrm{HER}$ on for $\mathrm{Pt}(111)$ at $\mathrm{pH} 1$ and $\mathrm{pH} 13$ and on a $\mathrm{Pt}(111)$ electrode decorated with $0.1 \mathrm{ML}$ of $\mathrm{Ni}(\mathrm{OH})_{2}$ in $0.1 \mathrm{M} \mathrm{NaOH}\left(\mathrm{pH} \mathrm{13)}\right.$, under argon atmosphere. The polarization curves were registered at a scan rate of $10 \mathrm{mV} . \mathrm{s}^{-1}$. b) The inverse of the charge transfer resistance as a function of potential for $\mathrm{Pt}(111)$ (black squares) and $\mathrm{Pt}(111) \mathrm{electrode}$ decorated with $\sim 10 \%$ of $\mathrm{Ni}(\mathrm{OH})_{2}$ in $0.1 \mathrm{M} \mathrm{NaOH}, \mathrm{pH} 13$ (blue squares); argon atmosphere. The data shows how the presence of $\mathrm{Ni}(\mathrm{OH})_{2}$ increases the rate of $\mathrm{H}$-UPD formation on $\mathrm{Pt}(111)$ in alkaline solution. The $\mathrm{R}_{\mathrm{ct}}$ was obtained from the fitting parameters for admittance plots (see Supplementary Figure S5).

\section{Laser-induced temperature jump measurements of solvent reorganization}


Our suggestion here is that the rate of hydrogen adsorption on $\mathrm{Pt}(111)$ (and hence of hydrogen evolution, if hydrogen adsorption is rate-determining) has a $\mathrm{pH}$ dependent barrier because the interfacial water structure at the $\mathrm{Pt}(111)$ electrode is $\mathrm{pH}$ dependent. During the hydrogen adsorption reaction, charge has to move through the interfacial double layer $\left(\mathrm{H}^{+}\right.$in acid and $\mathrm{OH}^{-}$in alkaline). The rate of such a charge transfer reaction depends on the extent to which the solvation environment (i.e. the water) can accommodate this charge migration. If the interfacial water is easily reorganized, charge transfer through the double layer will be rapid; if the interfacial water is rigid and difficult to reorganize, charge transfer through the double layer will be slow. An important parameter to influence the extent to which the interfacial water can be reorganized is the interfacial electric field. The interfacial electric field is determined by the charge separation between the metal electrode and the electrolyte solution, which in turn is dependent on the position of the potential of zero charge (pzc). In the presence of adsorption reactions, a distinction should be made between total and free charges ${ }^{38}$. While the latter represents the true charge determining the electric field at the interface, the total charge, which includes also the charge stored in the bond of adsorbed species, is the only available parameter from electrochemical measurements. To obtain the potential of zero free charge, additional measurements and assumptions are necessary to separate adsorption reactions from the true capacitive processes. In the absence of a strong interfacial electric field, i.e. close to the potential of zero (free) charge (pzfc), water is relatively free to reorient and hence easy to reorganize. In the presence of a strong interfacial electric field, i.e. further away from the pzfc, water is much more rigid and hence more difficult to reorganize. As has been shown by previous work ${ }^{39-41}$, while the pzfc cannot be directly measured, an estimation can be obtained from values of $\mathrm{CO}$ charge displacement experiments. This is achieved by extrapolating the charge in the double layer region either into the hydrogen adsorption region (acid solutions) or into the hydroxyl adsorption region (alkaline solutions). In this way, a relatively constant value of pzfc is obtained, independent of $\mathrm{pH}$ on the SHE scale. This means that pzfc changes from the double layer region ( $0.34 \mathrm{~V}_{\mathrm{RHE}}$ ) in acidic solutions to the onset of the surface oxidation (ca. 1.0 $\mathrm{V}_{\mathrm{RHE}}$ ) in strongly alkaline solutions ${ }^{40}$. As a consequence, at a given potential on the RHE scale, the strength of the electric field and the energy associated with the (re)organization of interfacial solvent molecules will depend on $\mathrm{pH}$. Since the H-UPD region is far from the pzfc of $\mathrm{Pt}(111)$ in alkaline media, the 
hydrogen adsorption reaction is slow in alkaline solutions. In this model, a way to compensate this strong electric field is to use surface modifiers, such as nickel, that bring the pzfc closer to the equilibrium potential of the hydrogen evolution reaction.

The estimation of the pzfc mentioned above is based on an extrapolation and therefore subject to some uncertainty, since the double layer capacity is likely to change when hydroxyl groups adsorb on the electrode surface. In order to evaluate the sign and magnitude of the electric field at the interface and the effect of $\mathrm{Ni}(\mathrm{OH})_{2}$ on them, we have employed the laser-induced temperature jump method. This technique uses short laser pulses to increase the temperature of the solvent at the interface for a very brief period of time ${ }^{42,43}$. During the relaxation at open circuit, the temporal change in potential induced by the temperature perturbation is registered. The sign of the transient gives information about the average orientation of the interfacial solvent that existed at the potential before the potentiostatic control was removed and the short laser pulse was applied. It is implied in this interpretation that adsorption processes are not fast enough to follow the temperature perturbation. Specifically, the potential for which the polarity of the transient changes is identified as the potential of maximum entropy (pme), which is expected to be closely related to the pzfc. Previous comparisons ${ }^{43}$ between the pme and pzfc of $\mathrm{Pt}(111)$ in acidic solution indeed show a close correspondence between the two quantities.

Details regarding the laser-induced temperature jump method can be found in the literature ${ }^{43}$, and a detailed study of the pme of $\mathrm{Pt}(111)$ in alkaline solutions will be given in a separate publication. In the present work we focus on the effect of $\mathrm{Ni}(\mathrm{OH})_{2}$ on the pme. The method was used to determine the pme of the $\mathrm{Pt}(111)$ interface at $\mathrm{pH}=10$ and $\mathrm{pH}=13$ (see Figures 4 and 5 , respectively), as well as the effect of a submonolayer amount of $\mathrm{Ni}(\mathrm{OH})_{2}$ deposited on the $\mathrm{Pt}(111)$ surface on the pme. At high $\mathrm{pH}$ values such as $\mathrm{pH}=13$, the interference of $\mathrm{OH}$ adsorption introduces uncertainty in the interpretation of the potential transients. At $\mathrm{pH}=10$, however, the concentration of hydroxyl in solution is low enough, so the $\mathrm{OH}$ coverage cannot follow the rapid temperature change. The results of the measurements for $\mathrm{pH}=10$ are shown in Figure 4. We present the cyclic voltammetries for the bare and decorated electrode in Figure 4a. The transients for $\mathrm{Pt}(111)$ at $\mathrm{pH}=10$ in Figure $4 \mathrm{~b}$ and $4 \mathrm{c}$ are negative in the whole potential range studied, i.e. there is no change of polarity that would indicate inversion of water dipoles. This is consistent with the location of the 
pzfc above the region of $\mathrm{OH}$ adsorption. Alternatively, it may happen that $\mathrm{OH}$ adsorption on the electrode surface may screen the positive charge on the electrode, orienting the water dipoles with the positive end towards the surface. A similar situation has been observed with other anions both on Pt and Au single crystals ${ }^{44,45}$.

To quantify the effect of $\mathrm{Ni}(\mathrm{OH})_{2}$ adsorption on the pzfc we calculate the thermal coefficient $(\partial E / \partial T)_{P, T, \mu_{i}, \Gamma_{i}}$ from the slope of the potential decay after the temperature jump. These are shown in figure $4 \mathrm{c}$ for the bare electrode and the electrode modified with $\mathrm{Ni}(\mathrm{OH})_{2}$. This comparison reveals that $\mathrm{Ni}(\mathrm{OH})_{2}$ causes a clear decrease in the thermal coefficient that can be interpreted in terms of a decrease of the electric field caused by a negative shift of the pzfc ${ }^{45}$. This shift in pme/pzfc is in accordance with our model for the enhancement of the H-UPD and hydrogen evolution by the presence of $\mathrm{Ni}(\mathrm{OH})_{2}$ on the surface as proposed above, relating the hydrogen adsorption and hydrogen evolution rates to the energy required for the water reorganization in the electrode interface. This result is in line with previous results that have demonstrated that electropositive adatoms significantly decrease the value of the pme and therefore the pzfc ${ }^{46}$, further supporting our interpretation. 
Figure 4. Cyclic voltammetry of $\mathrm{Pt}(111)$ in contact with the buffered solution with $\mathrm{pH}=10: 0.05 \mathrm{M} \mathrm{Na}_{2} \mathrm{CO}_{3}+0.05 \mathrm{M} \mathrm{NaHCO}_{3}$.
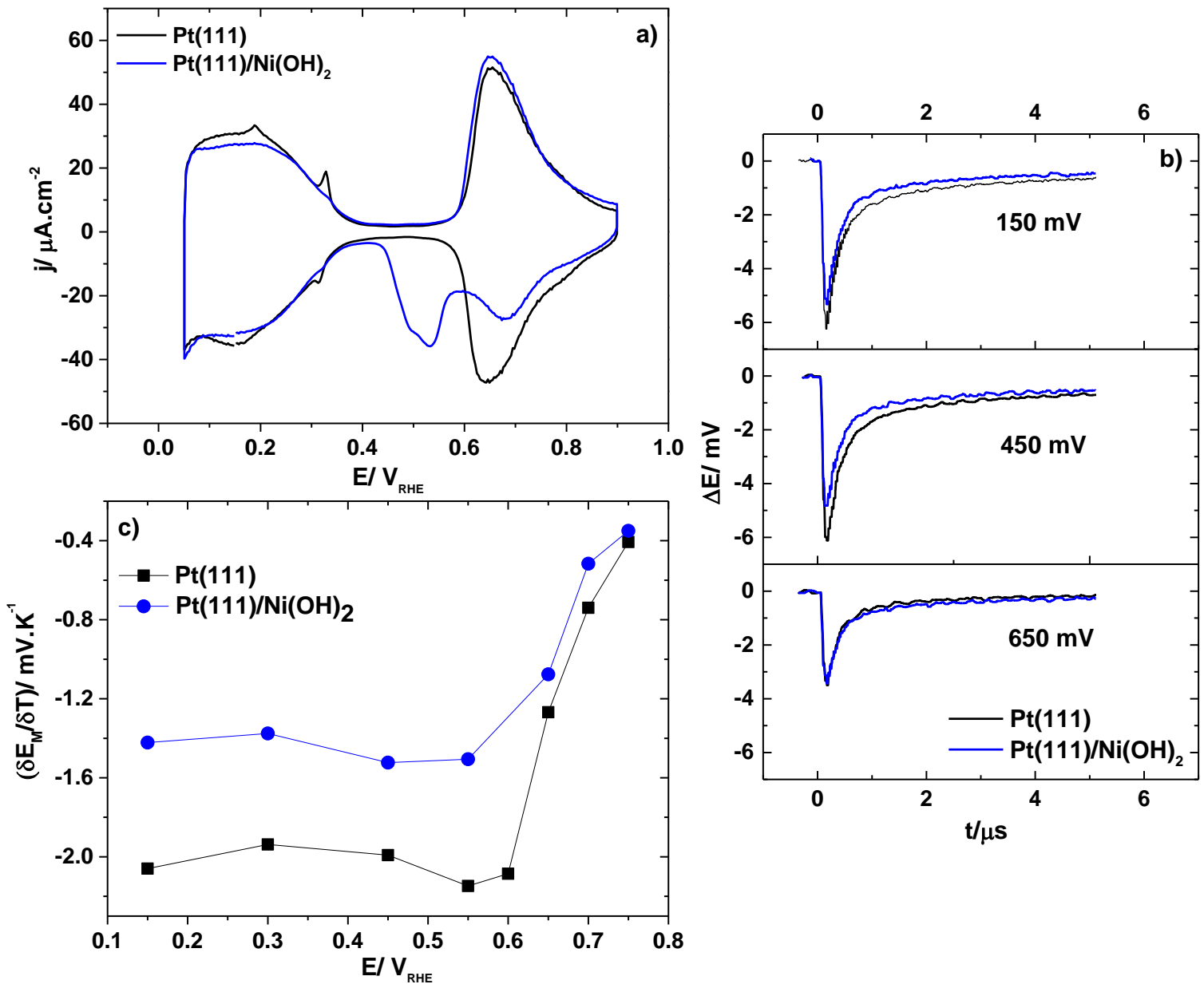

Black line: $\mathrm{Pt}(111)$; blue line: $\mathrm{Pt}(111)$ modified with a submonolayer amount of $\mathrm{Ni}(\mathrm{OH})_{2}(\sim 12 \%$ coverage). Scan rate: $50 \mathrm{mV} / \mathrm{s}$. b) Coulostatic laser transients at different applied potentials for $\mathrm{Pt}(111)$ and $\mathrm{Pt}(111)$ decorated with $\mathrm{Ni}(\mathrm{OH})_{2}$. c) Temperature coefficient of the double layer potential for: $\mathrm{Pt}(111)$ (black line), $\mathrm{Pt}(111) / \mathrm{Ni}(\mathrm{OH})_{2}$ (blue line). Beam energy: $1.0 \mathrm{~mJ}$. The difference between thermal coefficients of pristine $\mathrm{Pt}(111)$ and $\mathrm{Pt}(111) / \mathrm{Ni}(\mathrm{OH})_{2}$ at each applied potential is shown in Supplementary Figure s6.

The transients for $\mathrm{Pt}(111)$ and $\mathrm{Pt}(111) / \mathrm{Ni}(\mathrm{OH})_{2}$ at $\mathrm{pH}=13$ in Figure $5 \mathrm{~b}$ and $5 \mathrm{c}$ show negative transients at $0.525 \mathrm{~V}_{\mathrm{RHE}}$ and $0.550 \mathrm{~V}_{\mathrm{RHE}}$, respectively. At these potentials, the interfacial water molecules are oriented with their hydrogens pointing towards the electrode surface. We observe a change in polarity of the interfacial species at 0.700 $\mathrm{V}_{\mathrm{RHE}}$ for the bare $\mathrm{Pt}(111)$ and $0.675 \mathrm{~V}_{\mathrm{RHE}}$ for the decorated $\mathrm{Pt}(111)$. This may suggest that from this potential the water molecules have the hydrogens pointing away from the electrode surface. However, at $\mathrm{pH}=13$ it is less likely that we can ignore the effect of hydroxyl adsorption on the observed transients. If the observed change in polarity between the transients of the bare $\mathrm{Pt}(111)$ and the $\mathrm{Pt}(111) / \mathrm{Ni}(\mathrm{OH})_{2}$ is due to a 
change in orientation of interfacial water, that would indicate that the presence of $\mathrm{Ni}(\mathrm{OH})_{2}$ has shifted the pme ca. $25 \mathrm{mV}$ negatively with respect to the bare $\mathrm{Pt}(111)$.

We emphasize that the experiments in Figures 4 and 5 aim at showing a shift in the pme when $\mathrm{Pt}(111)$ in alkaline media is modified with a small amount of $\mathrm{Ni}(\mathrm{OH})_{2}$ (ca. $20 \%$ coverage), consistent with the idea that the presence of $\mathrm{Ni}(\mathrm{OH})_{2}$ changes the interfacial electric field. A detailed quantitative modelling of the transients is beyond the scope of the current manuscript.
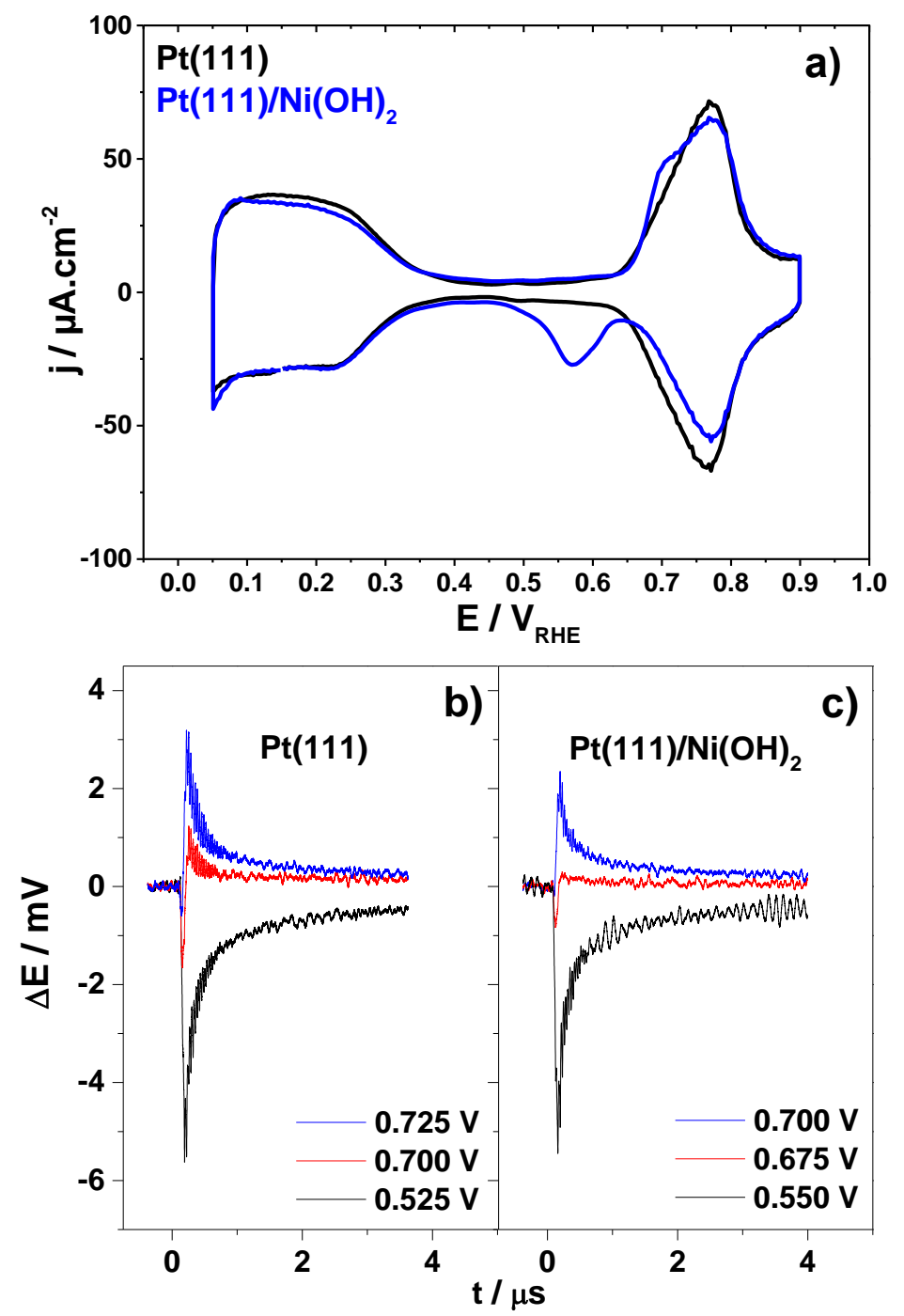

Figure 5. a) Cyclic voltammograms for $\mathrm{Pt}(111)$ in $0.1 \mathrm{M} \mathrm{NaOH}$ under argon atmosphere, registered at a scan rate of $50 \mathrm{mV} . \mathrm{s}^{-1}$ : The black line shows the blank of the bare electrode, whereas the blue line shows the $\operatorname{Pt}(111)$ electrode decorated with $\mathrm{Ni}(\mathrm{OH})_{2}$, growth with instant deposition from a solution containing $5 \mathrm{mM} \mathrm{Ni}\left(\mathrm{NO}_{3}\right)_{2}\left(\mathrm{Ni}^{0}\right)$ and 3 cycles in the $0.1 \mathrm{M} \mathrm{NaOH}$ solution $(\sim 13 \%$ coverage). A voltammogram of $\mathrm{Pt}(111)$ with a higher coverage of $\mathrm{Ni}(\mathrm{OH})_{2}$ is shown in Supplementary Figure S7 b) Laserinduced coulostatic potential transients collected for the $\mathrm{Pt}(111)$ electrode. c) Laser-induced coulostatic potential transients collected for the $\mathrm{Pt}(111)$ electrode decorated with $\mathrm{Ni}(\mathrm{OH})_{2}$. Beam energy: $1 \mathrm{~mJ}$. 
Our model for the role of interfacial solvent reorganization in the hydrogen adsorption reaction bears resemblance to a model for electrochemical proton transfer suggested several years ago by Pecina and Schmickler ${ }^{47}$. Using Monte Carlo simulations to sample water configurations near a negatively charged surface, as it is the case in alkaline solutions, they calculated the activation energy for proton transfer through the interfacial water layer. They showed that the free activation energy for proton transfer increases at high interfacial fields due to the effect referred to above, whereas at lower field, the activation energy in their simulations in fact lowers due to a favorable configurational effect. These simulations correlate well with the change in the pzfc of the $\mathrm{Pt}(111)$ in presence of small amounts of $\mathrm{Ni}(\mathrm{OH})_{2}$, since the presence of $\mathrm{Ni}(\mathrm{OH})_{2}$ lowers the interfacial electric field and thereby lowers the energetic barrier for the reorganization of the interfacial water network. The water in the work of Pecina and Schmickler was modeled by classical model potentials but it is not expected that the qualitative effect will change if more accurate potentials, such as provided by first-principles density functional theory calculations, would have been used. More recently, Rossmeisl et al. ${ }^{48,49}$ also considered models for the $\mathrm{pH}$ dependence of electrochemical proton transfer barriers, and emphasized the $\mathrm{pH}$ dependent contribution of the configurational entropy of the proton as the origin of the $\mathrm{pH}$ dependent kinetics. Our model highlights, on the other hand, the $\mathrm{pH}$ dependent enthalpy of interfacial water reorganization, as related to the $\mathrm{pH}$ dependent potential of zero free charge of $\mathrm{Pt}$ on the RHE scale. It is also important to emphasize that this model of interfacial solvent reorganization should apply equally to $\mathrm{H}$-UPD and $\mathrm{H}$ OPD, as it is independent of the final state of hydrogen on the platinum.

\section{Conclusions}

Given the experimental evidence presented in this paper, we conclude that a $\mathrm{pH}$ dependent shift in the $\mathrm{H}$-binding energy does not explain the significant $\mathrm{pH}$ dependence of the HER overpotential on $\mathrm{Pt}(111)$. We have studied its kinetic origin by a thorough interpretation of combined impedance measurements, Tafel plot analysis and laser-induced temperature jump experiments of interfacial solvent reorganization. We showed that not only the HER is slower in alkaline media, but also the H-UPD adsorption is slower, even if the thermodynamic driving force is the 
same on the RHE scale for the different $\mathrm{pH}$ values. Also the rate-determining step in the mechanism for the hydrogen evolution reaction is affected by the $\mathrm{pH}$, in agreement with the observation that hydrogen adsorption becomes a kinetically hindered step in alkaline media.

We explain our observations by a new model for the HER highlighting the role of interfacial water reorganization in the hydrogen adsorption step. This model is based on the experimental result that in acidic media, the H-UPD and HER occur close to the potential of zero charge, whereas in alkaline media, the H-UPD and the hydrogen evolution occur far from the potential of zero (total) charge. This would imply that in alkaline media the interfacial water network at the potential of H-UPD and HER interacts strongly with the strong interfacial electric field and is therefore more rigid and more difficult to reorganize during the charge transfer through the electrical double layer. This causes the hydrogen step to be slow in alkaline media. The presence of $\mathrm{Ni}(\mathrm{OH})_{2}$ in submonolayer amounts improves the $\mathrm{H}$-UPD charge transfer and the hydrogen evolution kinetics in alkaline $\mathrm{pH}$ by inducing a shift in the potential of total zero charge toward the HER equilibrium potential, as confirmed by laserinduced temperature jump experiments. In this new model, $\mathrm{Ni}(\mathrm{OH})_{2}$ promotes the hydrogen evolution reaction by lowering the energy barrier necessary for the reorganization of the interfacial water network, allowing a more efficient proton/hydroxide transfer through the double layer. Importantly, the new model also implies that the hydrogen binding energy is not the sole descriptor for the HER kinetics. The strength of the interfacial electric field, as determined by the distance of the potential of zero (total) charge (or the potential of maximum entropy) from the equilibrium potential of the reaction to be catalysed, impacts on the rate of hydrogen adsorption by influencing the energetic barrier associated with the reorganisation of interfacial water during charge transfer.

\section{Methods}

Experiments were carried out in a fluorinated ethylene propylene (FEP, Nalgene) electrochemical cell for alkaline solutions, whereas a glass cell was preferred for acidic solutions, using a three-electrode assembly at room temperature in both 
cases. The cells and glassware were initially cleaned by boiling in a 1:1 mixture of concentrated sulfuric acid and nitric acid, rinsed thoroughly and boiled with ultra clean water (Milipore ${ }^{\circledR}$ MiliQ; resistivity $>18.2 \mathrm{M} \Omega . \mathrm{cm}$ ) five times before each set of experiments. All the glassware and cells were kept in a permanganate/sulfuric acid mixture when not in use.

Solutions of different $\mathrm{pH}$ value were prepared as follows: for $\mathrm{pH} 1$ we used solutions of $0.1 \mathrm{M} \mathrm{HClO}_{4}$ for cyclic voltammetry and $0.12 \mathrm{M}$ for the Tafel slope polarization curves (Merck $70 \%$, Suprapur). In the preparation of $\mathrm{pH} 1.8$ we used a mixture of $0.01 \mathrm{M} \mathrm{HClO}_{4}+0.090 \mathrm{M} \mathrm{NaClO}_{4}$ (monohydrate, EMSURE), and for $\mathrm{pH} 2.5$ the mixture was $0.001 \mathrm{M} \mathrm{HClO}_{4}+0.099 \mathrm{M} \mathrm{NaClO}_{4}$. Phosphate buffer solutions of 0.1 $\mathrm{M}$ were prepared for $\mathrm{pH} 4.3,7,9.1$ and 9.8, using sodium dihydrogen phosphate and/or disodium hydrogen phosphate (Merck 99.99\%, Suprapur) and orthophosphoric acid (Merck 85\%, Suprapur) as indicated by the Henderson-Hesselbach equation for buffers. For $\mathrm{pH} 11$ and 12 we prepared solutions of $0.001 \mathrm{M} \mathrm{NaOH}$ (Sigma-Aldrich, 99.99\% trace metal basis) + 0.090 $\mathrm{M} \mathrm{NaClO}_{4}$, and $0.01 \mathrm{M} \mathrm{NaOH}+$ $0.090 \mathrm{M} \mathrm{NaClO}_{4}$ respectively. Solutions of $0.1 \mathrm{M} \mathrm{NaOH}$ were prepared for $\mathrm{pH} 13$.

Bead-type single crystal platinum electrodes with a (111) orientation $(<0.5$ degrees) were used as working electrodes (WE). Before each experiment the electrode was flame-annealed for $1 \mathrm{~min}$ and cooled down to room temperature in a 3:1 proportion $\mathrm{Ar}+\mathrm{H}_{2}$ mixture (Linde 6.0). The electrode was transferred to the electrochemical cell with a protective droplet of deoxygenated water. All measurements were carried out with the Pt single crystal electrode in a hanging meniscus configuration. A platinum wire was used as counter electrode (CE), and the reference electrode was an internal reversible hydrogen electrode (RHE). For the EIS measurements a home-made, self-contained hydrogen electrode was placed in the same electrolyte, with a Teflon connection in order to avoid contact between the solution and the glass. A $10 \mu \mathrm{F}$ capacitor was connected, as a noise filter, between the RE and a platinum wire immersed in the solution. All potentials noted are referred to the reversible hydrogen electrode (RHE).

The argon was bubbled through a $3 \mathrm{M} \mathrm{KOH}$ trap before it entered the cell, in order to remove impurities which may be present from the tubing. The cell was placed inside a Faraday cage. Cyclic voltammetries and impedance spectra were 
collected using a computer-controlled Ivium A06075 potentiostat (Iviumstat). Impedance spectra were measured with frequencies ranging from $10 \mathrm{kHz}$ to $0.1 \mathrm{~Hz}$ and a peak-to-peak amplitude of $5 \mathrm{mV}$. The data was fitted to the equivalent electric circuit (EEC) shown in 2a, using a Python script, employing the Levenberg-Marquardt algorithm for non-linear least square regression. All shown impedance data were verified to satisfy Kramers-Kronig relations.

For the deposition of a submonolayer of $\mathrm{Ni}(\mathrm{OH})_{2}$ on the $\mathrm{Pt}(111)$ electrode we used a solution of $0.1 \mathrm{M} \mathrm{NaClO}_{4}$ and $5 \mathrm{mM} \mathrm{Ni}\left(\mathrm{NO}_{3}\right)_{2}$ (Sigma-Aldrich $99.999 \%$, trace metals basis). The solution was purged with Argon for 10 minutes and the $\operatorname{Pt}(111)$ electrode was modified via instantaneous electroless deposition, by placing the electrode in the deaerated solution for $10 \mathrm{~s}$. The coverage of the nickel hydroxide formed, expressed as percentage, is reported as the normalized difference between the H-UPD region of the blank and the H-UPD region of the electrode decorated with nickel hydroxide. After deposition, the $\mathrm{Pt}(111)-\mathrm{Ni}$ electrode was rinsed with ultrapure water and brought to hanging meniscus configuration in a cell containing a $0.1 \mathrm{M}$ $\mathrm{NaOH}$ solution, in order to perform cyclic voltammetry (3 cycles). The laser induced temperature jump experiment was performed as described elsewere ${ }^{50}$. Pulses of $5 \mathrm{~ns}$ of the second harmonic of a Nd-YAG laser (532 nm) were used as laser source, with an energy density around $8 \mathrm{~mJ}^{-\mathrm{cm}^{-2}}$ (the laser beam diameter is $4 \mathrm{~mm}$ and the energy of the laser pulse is $1 \mathrm{~mJ}$ ), a value of energy small enough to prevent the damage of the $\mathrm{Ni}(\mathrm{OH})_{2}$ deposit on the $\mathrm{Pt}(111)$ electrode. The laser energy was measured with a pyroelectric sensor head (Model M-935-10). The only effect from laser irradiation is the increase of the temperature of the interface.

\section{Acknowledgements}

This work was supported by a TOP grant from the Netherlands Organization for Scientific Research (NWO). Support from MINECO (Spain) through project CTQ2013-44083-P is acknowledged.

\section{Author contributions}


I.L-Y., V.C., J.M.F., and M.T.M.K. designed the experiments. I.L-Y. and W.D.Z.W. carried out the electrochemical experiments. I.L-Y. and P.S-P. carried out the laserinduced temperature-jump experiments. I.L-Y. and M.T.M.K. co-wrote the manuscript and all authors edited the manuscript.

\section{References}

1 Goldemberg, J. Ethanol for a Sustainable Energy Future. Science 315, 808810, doi:10.1126/science.1137013 (2007).

2 Hoffert, M. I. et al. Advanced Technology Paths to Global Climate Stability: Energy for a Greenhouse Planet. Science 298, 981-987, doi:10.1126/science.1072357 (2002).

3 Turner, J. A. Sustainable Hydrogen Production. Science 305, 972-974, doi:10.1126/science.1103197 (2004).

4 Bockris, J. O. M. The origin of ideas on a Hydrogen Economy and its solution to the decay of the environment. International Journal of Hydrogen Energy 27, 731-740, doi:http://dx.doi.org/10.1016/S0360-3199(01)00154-9 (2002).

5 Kudo, A. \& Miseki, Y. Heterogeneous photocatalyst materials for water splitting. Chemical Society Reviews 38, 253-278, doi:10.1039/B800489G (2009).

$6 \mathrm{Ni}$, M., Leung, M. K. H., Leung, D. Y. C. \& Sumathy, K. A review and recent developments in photocatalytic water-splitting using for hydrogen production. Renewable and Sustainable Energy Reviews 11, 401-425, doi:http://dx.doi.org/10.1016/j.rser.2005.01.009 (2007).

7 Van Hook, J. P. Methane-Steam Reforming. Catalysis Reviews 21, 1-51, doi:10.1080/03602458008068059 (1980).

8 Barnhart, C. J. \& Benson, S. M. On the importance of reducing the energetic and material demands of electrical energy storage. Energy \& Environmental Science 6, 1083-1092, doi:10.1039/C3EE24040A (2013).

9 H. Hashemi, S. M., Modestino, M. A. \& Psaltis, D. A membrane-less electrolyzer for hydrogen production across the $\mathrm{pH}$ scale. Energy \& Environmental Science 8, 2003-2009, doi:10.1039/C5EE00083A (2015). 
10 Huang, Q., Ye, Z. \& Xiao, X. Recent progress in photocathodes for hydrogen evolution. Journal of Materials Chemistry A 3, 15824-15837, doi:10.1039/C5TA03594E (2015).

11 Man, I. C. et al. Universality in Oxygen Evolution Electrocatalysis on Oxide Surfaces. ChemCatChem 3, 1159-1165, doi:10.1002/cctc.201000397 (2011).

12 Calle-Vallejo, F., Díaz-Morales, O. A., Kolb, M. J. \& Koper, M. T. M. Why Is Bulk Thermochemistry a Good Descriptor for the Electrocatalytic Activity of Transition Metal Oxides? ACS Catalysis 5, 869-873, doi:10.1021/cs5016657 (2015).

13 Diaz-Morales, O., Ledezma-Yanez, I., Koper, M. T. M. \& Calle-Vallejo, F. Guidelines for the Rational Design of Ni-Based Double Hydroxide Electrocatalysts for the Oxygen Evolution Reaction. ACS Catalysis 5, 53805387, doi:10.1021/acscatal.5b01638 (2015).

14 Friebel, D. et al. Identification of Highly Active Fe Sites in ( $\mathrm{Ni}, \mathrm{Fe}) \mathrm{OOH}$ for Electrocatalytic Water Splitting. Journal of the American Chemical Society 137, 1305-1313, doi:10.1021/ja511559d (2015).

15 Bockris, J. O. M. \& Potter, E. C. The Mechanism of the Cathodic Hydrogen Evolution Reaction. Journal of The Electrochemical Society 99, 169-186, doi:10.1149/1.2779692 (1952).

16 Conway, B. E. \& Bai, L. State of adsorption and coverage by overpotentialdeposited $\mathrm{H}$ in the $\mathrm{H} 2$ evolution reaction at $\mathrm{Au}$ and Pt. Electrochimica Acta 31, 1013-1024, doi:http://dx.doi.org/10.1016/0013-4686(86)80017-2 (1986).

17 Schouten, K. J. P., van der Niet, M. J. T. C. \& Koper, M. T. M. Impedance spectroscopy of $\mathrm{H}$ and $\mathrm{OH}$ adsorption on stepped single-crystal platinum electrodes in alkaline and acidic media. Physical Chemistry Chemical Physics 12, 15217-15224, doi:10.1039/COCP00104J (2010).

18 Subbaraman, R. et al. Enhancing Hydrogen Evolution Activity in Water Splitting by Tailoring $\mathrm{Li}+-\mathrm{Ni}(\mathrm{OH}) 2-\mathrm{Pt}$ Interfaces. Science 334, 1256-1260 (2011).

19 Danilovic, N. et al. Enhancing the Alkaline Hydrogen Evolution Reaction Activity through the Bifunctionality of $\mathrm{Ni}(\mathrm{OH}) 2 /$ Metal Catalysts. Angewandte Chemie 124, 12663-12666, doi:10.1002/ange.201204842 (2012).

20 Strmcnik, D. et al. Improving the hydrogen oxidation reaction rate by promotion of hydroxyl adsorption. Nat Chem 5, 300-306, 
doi:http://www.nature.com/nchem/journal/v5/n4/abs/nchem.1574.html\#supple mentary-information (2013).

21 Sheng, W., Gasteiger, H. A. \& Shao-Horn, Y. Hydrogen Oxidation and Evolution Reaction Kinetics on Platinum: Acid vs Alkaline Electrolytes. Journal of The Electrochemical Society 157, B1529-B1536, doi:10.1149/1.3483106 (2010).

22 Sheng, W. et al. Correlating hydrogen oxidation and evolution activity on platinum at different $\mathrm{pH}$ with measured hydrogen binding energy. Nature communications 6, 5848, doi:10.1038/ncomms6848 (2015).

23 Greeley, J., Jaramillo, T. F., Bonde, J., Chorkendorff, I. \& Norskov, J. K. Computational high-throughput screening of electrocatalytic materials for hydrogen evolution. Nat Mater 5, 909-913, doi:http://www.nature.com/nmat/journal/v5/n11/suppinfo/nmat1752_S1.html (2006).

24 Subbaraman, $R$. et al. Trends in activity for the water electrolyser reactions on 3d $\mathrm{M}(\mathrm{Ni}, \mathrm{Co}, \mathrm{Fe}, \mathrm{Mn})$ hydr(oxy)oxide catalysts. Nat Mater 11, 550-557, doi:http://www.nature.com/nmat/journal/v11/n6/abs/nmat3313.html\#suppleme ntary-information (2012).

25 Laursen, A. B. et al. Electrochemical Hydrogen Evolution: Sabatier's Principle and the Volcano Plot. Journal of Chemical Education 89, 1595-1599, doi:10.1021/ed200818t (2012).

26 Skúlason, E. et al. Modeling the Electrochemical Hydrogen Oxidation and Evolution Reactions on the Basis of Density Functional Theory Calculations. The Journal of Physical Chemistry C 114, 18182-18197, doi:10.1021/jp1048887 (2010).

27 Durst, J. et al. New insights into the electrochemical hydrogen oxidation and evolution reaction mechanism. Energy \& Environmental Science 7, 22552260, doi:10.1039/C4EE00440J (2014).

28 van der Niet, M. J. T. C., Garcia-Araez, N., Hernández, J., Feliu, J. M. \& Koper, M. T. M. Water dissociation on well-defined platinum surfaces: The electrochemical perspective. Catalysis Today 202, 105-113, doi:http://dx.doi.org/10.1016/j.cattod.2012.04.059 (2013).

29 Morin, S., Dumont, H. \& Conway, B. E. Evaluation of the effect of twodimensional geometry of pt single-crystal faces on the kinetics of upd of $h$ 
using impedance spectroscopy. Journal of Electroanalytical Chemistry $\mathbf{4 1 2}$, 39-52, doi:http://dx.doi.org/10.1016/0022-0728(96)04612-8 (1996).

30 Gisbert, R., García, G. \& Koper, M. T. M. Adsorption of phosphate species on poly-oriented $\mathrm{Pt}$ and $\mathrm{Pt}\left(\begin{array}{lll}1 & 1 & 1\end{array}\right)$ electrodes over a wide range of $\mathrm{pH}$. $\begin{array}{llll}\text { Electrochimica } & \text { Acta } & \text { 55, }\end{array}$ doi:http://dx.doi.org/10.1016/j.electacta.2010.04.009 (2010).

31 Oelgeklaus, R., Rose, J. \& Baltruschat, H. On the rate of hydrogen and iodine adsorption on polycrystalline $\mathrm{Pt}$ and $\mathrm{Pt}(111)$. Journal of Electroanalytical Chemistry 376, 127-133, doi:http://dx.doi.org/10.1016/0022-0728(94)03439-7 (1994).

32 Schuldiner, S. Hydrogen Overvoltage on Bright Platinum: II . pH and Salt Effects in Acid, Neutral, and Alkaline Solutions. Journal of The Electrochemical Society 101, 426-432, doi:10.1149/1.2781294 (1954).

33 Conway, B. E. \& Bai, L. Determination of adsorption of OPD H species in the cathodic hydrogen evolution reaction at $\mathrm{Pt}$ in relation to electrocatalysis. Journal of Electroanalytical Chemistry and Interfacial Electrochemistry 198, 149-175, doi:http://dx.doi.org/10.1016/0022-0728(86)90033-1 (1986).

34 Shinagawa, T., Garcia-Esparza, A. T. \& Takanabe, K. Insight on Tafel slopes from a microkinetic analysis of aqueous electrocatalysis for energy conversion. Scientific Reports 5, 13801, doi:10.1038/srep13801 (2015).

35 Seto, K., lannelli, A., Love, B. \& Lipkowski, J. The influence of surface crystallography on the rate of hydrogen evolution at Pt electrodes. Journal of Electroanalytical Chemistry and Interfacial Electrochemistry 226, 351-360, doi:http://dx.doi.org/10.1016/0022-0728(87)80057-8 (1987).

36 Zheng, J., Yan, Y. \& Xu, B. Correcting the Hydrogen Diffusion Limitation in Rotating Disk Electrode Measurements of Hydrogen Evolution Reaction Kinetics. Journal of The Electrochemical Society 162, F1470-F1481, doi:10.1149/2.0501514jes (2015).

37 Hoshi, N., Asaumi, Y., Nakamura, M., Mikita, K. \& Kajiwara, R. Structural Effects on the Hydrogen Oxidation Reaction on $n(111)-(111)$ Surfaces of Platinum. The Journal of Physical Chemistry C 113, 16843-16846, doi:10.1021/jp9076239 (2009).

38 Climent, V., García-Araez, N., Herrero, E. \& Feliu, J. Potential of zero total charge of platinum single crystals: A local approach to stepped surfaces 
vicinal to Pt(111). Russian Journal of Electrochemistry 42, 1145-1160, doi:10.1134/s1023193506110012 (2006).

39 Garcia-Araez, N., Climent, V., Herrero, E., Feliu, J. M. \& Lipkowski, J. Thermodynamic approach to the double layer capacity of a $\operatorname{Pt}\left(\begin{array}{lll}1 & 1 & 1\end{array}\right)$ electrode in perchloric acid solutions. Electrochimica Acta 51, 3787-3793, doi:http://dx.doi.org/10.1016/j.electacta.2005.10.043 (2006).

40 Rizo, R., Sitta, E., Herrero, E., Climent, V. \& Feliu, J. M. Towards the understanding of the interfacial $\mathrm{pH}$ scale at $\mathrm{Pt}\left(\begin{array}{lll}1 & 1 & 1\end{array}\right)$ electrodes.

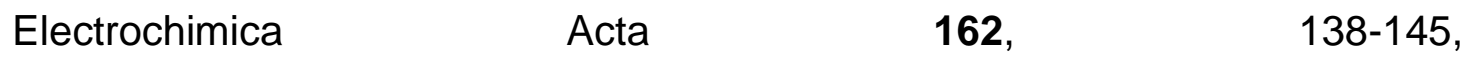
doi:http://dx.doi.org/10.1016/j.electacta.2015.01.069 (2015).

41 Cuesta, A. Measurement of the surface charge density of CO-saturated $\operatorname{Pt}(11$ 1) electrodes as a function of potential: the potential of zero charge of $\operatorname{Pt}(11$ 1). Surface Science 572 11-22, doi:http://dx.doi.org/10.1016/j.susc.2004.08.014 (2004).

42 Sebastián, P., Sandoval, A. P., Climent, V. \& Feliu, J. M. Study of the interface $\mathrm{Pt}(111) /$ [Emmim][NTf2] using laser-induced temperature jump experiments. Electrochemistry Communications 55, 39-42, doi:http://dx.doi.org/10.1016/j.elecom.2015.03.012 (2015).

43 Garcia-Araez, N., Climent, V. \& Feliu, J. Potential-Dependent Water Orientation on $\mathrm{Pt}(111), \mathrm{Pt}(100)$, and $\mathrm{Pt}(110)$, As Inferred from Laser-Pulsed Experiments. Electrostatic and Chemical Effects. The Journal of Physical Chemistry C 113, 9290-9304, doi:10.1021/jp900792q (2009).

44 Climent, V., Coles, B. A. \& Compton, R. G. Coulostatic Potential Transients Induced by Laser Heating of a $\mathrm{Pt}(111)$ Single-Crystal Electrode in Aqueous Acid Solutions. Rate of Hydrogen Adsorption and Potential of Maximum Entropy. The Journal of Physical Chemistry B 106, 5988-5996, doi:10.1021/jp020785q (2002).

45 Climent, V., Coles, B. A. \& Compton, R. G. Laser-Induced Potential Transients on a $\mathrm{Au}(111)$ Single-Crystal Electrode. Determination of the Potential of Maximum Entropy of Double-Layer Formation. The Journal of Physical Chemistry B 106, 5258-5265, doi:10.1021/jp020054q (2002).

46 García-Aráez, N., Climent, V. \& Feliu, J. M. Evidence of Water Reorientation on Model Electrocatalytic Surfaces from Nanosecond-Laser-Pulsed 
Experiments. Journal of the American Chemical Society 130, 3824-3833, doi:10.1021/ja0761481 (2008).

47 Pecina, O. \& Schmickler, W. A model for electrochemical proton-transfer reactions. Chemical Physics 228, 265-277, doi:http://dx.doi.org/10.1016/S0301-0104(97)00299-1 (1998).

48 Rossmeisl, J., Chan, K., Skúlason, E., Björketun, M. E. \& Tripkovic, V. On the $\mathrm{pH}$ dependence of electrochemical proton transfer barriers. Catalysis Today 262, 36-40, doi:http://dx.doi.org/10.1016/j.cattod.2015.08.016 (2016).

49 Rossmeisl, J., Chan, K., Ahmed, R., Tripkovic, V. \& Bjorketun, M. E. pH in atomic scale simulations of electrochemical interfaces. Physical Chemistry Chemical Physics 15, 10321-10325, doi:10.1039/C3CP51083B (2013).

50 Climent, V., Garcia-Araez, N., Compton, R. G. \& Feliu, J. M. Effect of Deposited Bismuth on the Potential of Maximum Entropy of $\mathrm{Pt}(111)$ SingleCrystal Electrodes. The Journal of Physical Chemistry B 110, 21092-21100, doi:10.1021/jp061982i (2006). 